\title{
La búsqueda de resistencia al tizón tardío en papas silvestres
}

\author{
Searching for late blight resistance in wild potatoes
}

\begin{abstract}
Guillermo Andrés Enciso-Maldonado ${ }^{1 *} \odot$, Héctor Lozoya-Saldaña ${ }^{1}{ }^{\oplus}$, Gabriela Díaz-García ${ }^{2}$, $^{\circ}$ Alfredo López-Salazar ${ }^{2}{ }^{1}$ Universidad Autónoma Chapingo. Instituto de Horticultura. Carretera MéxicoTexcoco, Chapingo, Estado de México. C. P. $56230{ }^{2}$ Universidad Autónoma Chapingo. Departamento de Parasitología Agrícola. Carretera México-Texcoco, Chapingo, Estado de México. C. P. 56230, México.

*Autor de correspondencia: gui77eenciso@gmail.com.
\end{abstract}

Recibido: 14 setiembre $2021 \quad$ Aceptado: 18 noviembre 2021

Recibido en versión modificada: 18 noviembre 2021

Enciso-Maldonado, G. A., Lozoya-Saldaña, H., DíazGarcía, G. \& López-Salazar, A. (2021). La búsqueda de resistencia al tizón tardío en papas silvestres. Investigaciones y Estudios - UNA, 12 (2), 36-47.

https://doi.org/10.47133/IEUNA2124b

Resumen. La papa (Solanum tuberosum L.) es la hortaliza más cultivada en el mundo. El principal factor limitante a nivel mundial es el tizón tardío (Phytophthora infestans), causando hasta pérdidas totales de producción. Una parte importante del mejoramiento genético de la papa se centra en la obtención de cultivares resistentes a esta enfermedad. Sin embargo, el patógeno es capaz de producir descendencias a partir de la reproducción sexual, generando nuevas cepas más virulentas, capaces de vencer la resistencia genética en nuevos cultivares. Por mucho tiempo, los genes de resistencia vertical de Solanum demissum han sido utilizados para la obtención de variedades resistentes, pero con éxito limitado debido a que son raza-específicos y porque el patógeno sobrepasa la inmunidad en poco tiempo. Actualmente existe un interés en el aprovechamiento de genes de resistencia horizontal o cuantitativa de diversas especies silvestres de Solanum, las cuales son difíciles de introducir a través de cruzamientos debido a la incompatibilidad de ploidías y al número de balance del endospermo (NBE). No obstante, algunas técnicas como la hibridación somática, la minería de alelos y el mejoramiento asistido por marcadores genéticos han permitido vencer estas barreras. De aquí la importancia de explorar e identificar nuevos genes de resistencia en las papas silvestres.

Palabras clave. Phytophthora infestans, Solanum tuberosum, recursos fitogenéticos.

Abstract. Potatoes (Solanum tuberosum L.) are the most cultivated vegetables in the world. The main limiting factor worldwide is late blight, a disease caused by the oomycete Phytophthora infestans, causing up even total production losses. An important part of potato genetic improvement focuses on obtaining cultivars resistant to this disease. However, the pathogen can produce offspring from sexual reproduction, generating new 
strains that are more virulent, resistant to fungicides and that allow it to overcome genetic resistance in new cultivars. For a long time, the vertical resistance genes of Solanum demissum were used to obtain resistant varieties, but their success have been limited by being race-specific and of shortterm duration. Currently there is an interest in horizontal or quantitative resistance genes of various wild species of Solanum, which are difficult to introduce due to ploidy incompatibility and to the Endosperm Balance Number (EBN). Nevertheless, techniques like somatic hybridization, allele mining and marker-assisted breeding have made possible to overcome those barriers. Hence the importance of exploring and identifying new resistance genes in wild species.

Keywords. Phytophthora infestans, Solanum tuberosum, plant genetic resources.

\section{INTRODUCCIÓN}

La papa (Solanum tuberosum), originaria la zona andina de Perú, Bolivia y Chile, es la hortaliza más cultivada del mundo y el cuarto alimento más producido después del trigo, el maíz y el arroz. La producción mundial alcanza 370.436 .581 toneladas en 17.340.986 hectáreas, que representa unos 80 billones de dólares (FAOSTAT, 2021). Por miles de años formó parte de la dieta humana y actualmente es un alimento básico para 1,3 mil millones de personas (Stokstad, 2019). En países desarrollados la ingesta de papas varía entre 50 y 150 g diarios, mientras que en los países en desarrollo varía entre 300 y 800 g diarios. Es una hortaliza rica en carbohidratos, proteínas, vitaminas C y B6, potasio y está recomendada para combatir la diabetes, cáncer y enfermedades cardiovasculares (Burgos et al., 2020).

Para satisfacer la demanda mundial, la papa se ha introducido a nuevos y diversos suelos y climas (Stokstad, 2019). Los países desarrollados eran los mayores productores de papa, sin embargo, desde 2005 los países en desarrollo pasaron a ser los líderes de la producción mundial (Devaux et al., 2020).

El mejoramiento genético de la papa es fundamental para mejorar la producción y cubrir la demanda. Los mayores esfuerzos del mejoramiento se centran en la resistencia al tizón tardío. Esta enfermedad es la principal amenaza mundial de la papa y puede destruir campos enteros de producción (Grunwald \& Flier, 2005). Su primera aparición fue en la década de 1840, devastando campos de papa en Irlanda, acontecimiento que terminó causando la muerte de un millón de personas y la migración de otros 1,5 millones (Haverkort et al., 2008). En años recientes se registraron epidemias en Estados Unidos y Canadá (Fry et al., 2015a), Europa (Schepers et al., 2015), Sudamérica (Lindqvist-Kreuze et al., 2020), África y Asia (Fry et al., 2015b).

Durante más de 100 años se trabajó con cruzamientos intra e interespecíficos para la obtención de materiales resistentes al tizón tardío, pero logrando sólo éxitos temporales. El potencial genético de las papas silvestres (Solanum spp.) aún es desconocido, pero en ellas ya se identificaron genes de resistencia al tizón tardío (Zoteyeva, 2020; Zoteyeva et al., 2012). El interés actual consiste en utilizar genes de resistencia cuantitativa de papas silvestres. 


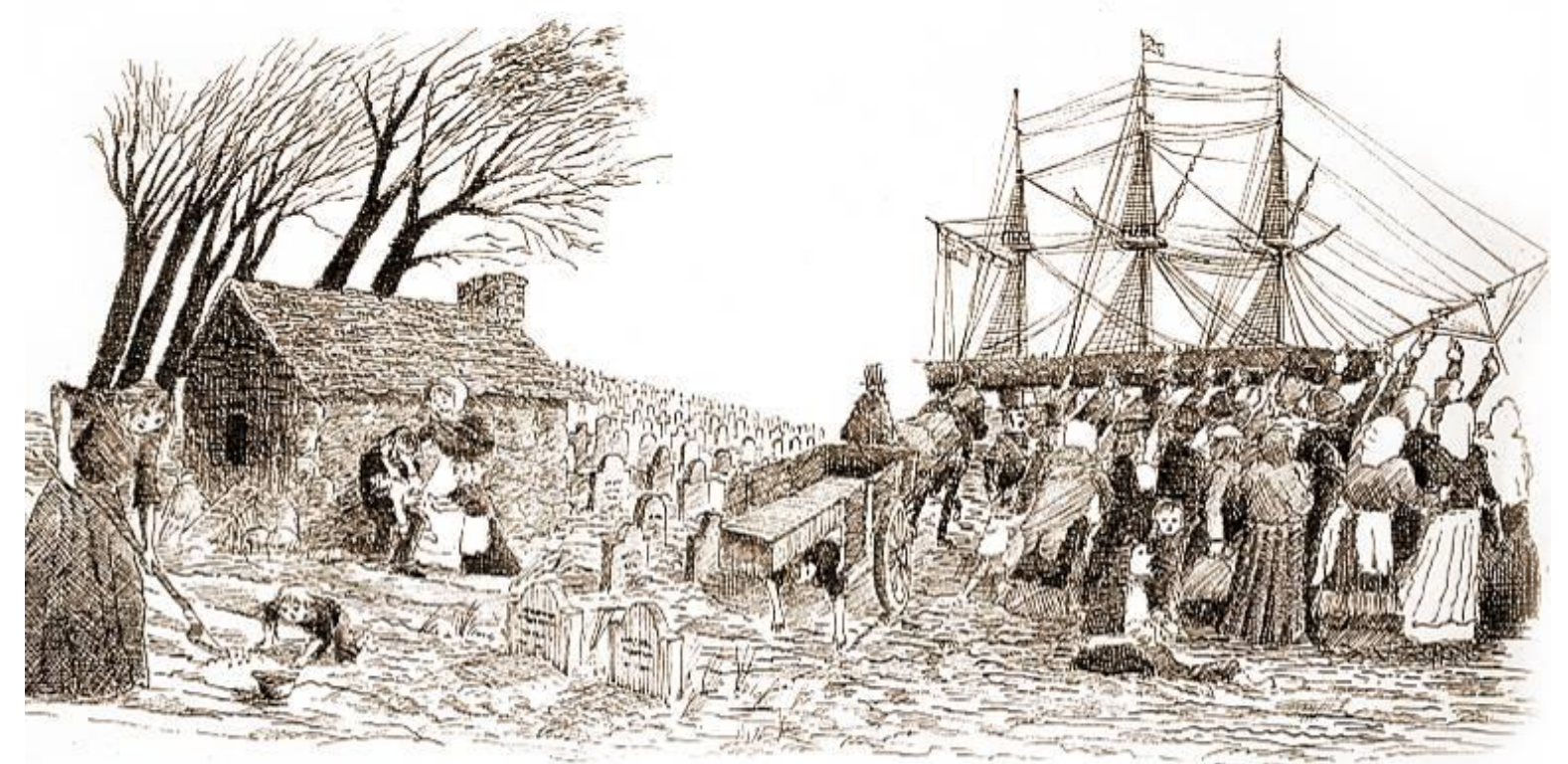

Figura 1. Alegoría de la hambruna irlandesa (1845-1850). Desde la pérdida de las cosechas de papa por el tizón tardío (izquierda) hasta la migración hacia el resto del mundo (derecha), principalmente a Europa continental, Estados Unidos y Australia (Cortesía del Programa Internacional Cooperativo del Tizón Tardío de la Papa (PICTIPAPA, A. C., México).

El objetivo de esta revisión es describir la importancia del tizón tardío a nivel internacional y los esfuerzos realizados en el mejoramiento genético para combatirlo dentro y fuera de México, dando énfasis a las papas silvestres como fuente de resistencia.

El infame Phytophthora infestans. El tizón tardío de la papa es causado por el hongo fitopatógeno $P$. infestans (Mont.) de Bary. Este patógeno ha sido calificado como "infame" por su papel en la hambruna irlandesa en la década de 1840 (Leesutthiphonchai et al., 2018), considerada la primera epidemia severa de plantas (Figura 1).

En ese entonces, la población irlandesa era altamente dependiente del monocultivo de papa y contaban con pocas variedades, lo que favoreció la emergencia de esta epidemia (Corredor-Moreno \& Saunders, 2020).

$P$. infestans es un oomiceto, hemibiotrófico que se reproduce asexual y sexualmente (Figura 2). En la fase asexual produce el esporangio, el cual se dispersa mediante el aire para alcanzar los órganos aéreos del hospedero, germina sobre ese tejido con temperaturas mayores a $21{ }^{\circ} \mathrm{C} \quad$ (siendo la temperatura óptima $25^{\circ} \mathrm{C}$ ) en un periodo de entre 8 y 48 horas (Foolad et al., 2008). Con temperaturas menores a $21{ }^{\circ} \mathrm{C}$ los esporangios liberan zoosporas biflageladas que le permiten moverse en el agua de los tejidos o en el suelo. Cuando entran en contacto con el hospedero pierden los flagelos y enquistan para producir tubos germinativos que sirven para invadir el tejido (Leesutthiphonchai et al., 2018). La esporulación óptima ocurre con $90 \%$ de humedad relativa (HR) y 12 a $18{ }^{\circ} \mathrm{C}$; una lesión produce hasta 300.000 esporangios por día (Fry \& Goodwin, 1997) y el ciclo de vida termina en cuatro días. En esta fase, los esporangios no tienen mecanismos de 


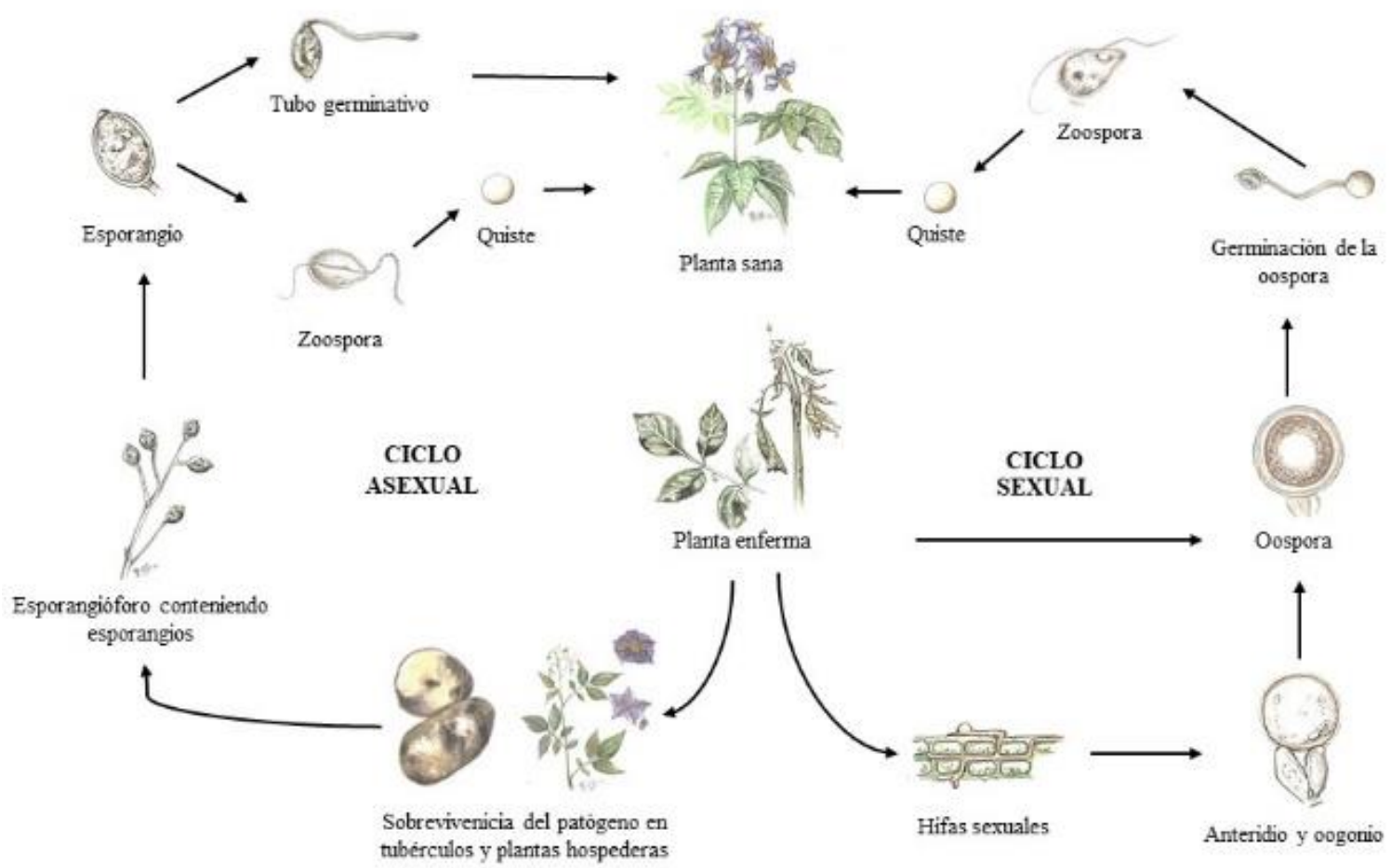

Figura 2. Ciclo de vida de Phytophthora infestans.

supervivencia en el largo plazo; para sobrevivir infectan tubérculos de papa, permitiendo la viabilidad del patógeno entre temporadas (Fry \& Grünwald, 2010). Por otro lado, la reproducción sexual en $P$. infestans es un fenómeno complejo que implica el intercambio hormonal entre los talos de aislados con grupos de apareamiento distintos, los cuales son denominados A1 y A2 (Mabon et al., 2021; Qi et al., 2005). El intercambio hormonal estimula la diferenciación de anteridios y oogonios, estructuras que producen los gametos sexuales masculinos y femeninos, respectivamente, que cuando se recombinan se reproducen sexualmente y dan lugar a la formación de oosporas grandes, de paredes gruesas, que le permiten sobrevivir fuera del hospedero (en restos de cultivo o en el suelo), convirtiéndose en fuente de inóculo primario para iniciar nuevas infecciones en la siguiente temporada (Fernández-Pavía et al., 2004; Lozoya-Saldaña, 2005; Fry \& Grünwald, 2010).
El centro de origen de $P$. infestans es el Valle de Toluca, ubicado en el altiplano mexicano y considerado el principal centro de diversidad del patógeno y el segundo mayor centro de diversidad de Solanum spp. (Grünwald \& Flier, 2005). En el Valle, durante el verano, diariamente la temperatura oscila entre 10 y $25^{\circ} \mathrm{C}$, la HR es superior al $90 \%$ al menos por 10 horas durante varios días y existe alternancia de períodos luminosidad/nubosidad; condiciones que son óptimas para el desarrollo del tizón (Lozoya-Saldaña, 2005). Además, están presentes hospederos con diversos grados de susceptibilidad y los tipos de compatibilidad del patógeno A1 y A2 se encuentran en proporción 1:1, permitiendo que las poblaciones del patógeno sean derivadas sexualmente con alta recombinación y amplia diversidad genética (Flier et al., 2003).

Actualmente, $P$. infestans es considerado un patógeno re-emergente debido a que sus poblaciones varían constantemente en todo el 
mundo, provocando la devastación de cultivos en lugares nuevos con una intensidad mayor en pocos días (Fry et al., 2015a; Vleeshouwers et al., 2011).

El éxito de este patógeno también se atribuye a su capacidad de sobrepasar la inmunidad del hospedero, a su esporulación profusa con un periodo latente corto que permite una rápida diseminación y una estructura del genoma que promueve su evolución adaptativa (Leesutthiphonchai et al., 2018).

Tipos de resistencia en papas. La resistencia al tizón tardío puede ser vertical u horizontal. La resistencia vertical presenta un alto grado de resistencia, pero a pocas cepas del patógeno, está mediada por uno o pocos genes $R$ que son razaespecíficos, de herencia mendeliana y son fáciles de incorporar para la obtención de nuevos genotipos resistentes. Por su parte, la resistencia horizontal es poligénica e inespecífica y protege a la planta contra varias cepas del patógeno; este tipo de resistencia es difícil de utilizar en programas de mejoramiento genético, pero es la más deseable por su potencial durabilidad (Karki et al., 2021; Lozoya-Saldaña, 2011). Por otro lado, la resistencia foliar difiere de la resistencia del tubérculo debido a que los genes involucrados en ambos órganos son distintos (Lal et al., 2013; Halterman et al., 2008; Kirk et al, 2001). Las evaluaciones de resistencia en el follaje muestran una respuesta consistente en diferentes ambientes y años, mientras que la respuesta observada en los tubérculos muestra una mayor variación, por ello es preferible evaluar la resistencia foliar para seleccionar genotipos resistentes (Mayton et al., 2010; Douches et al., 2002).

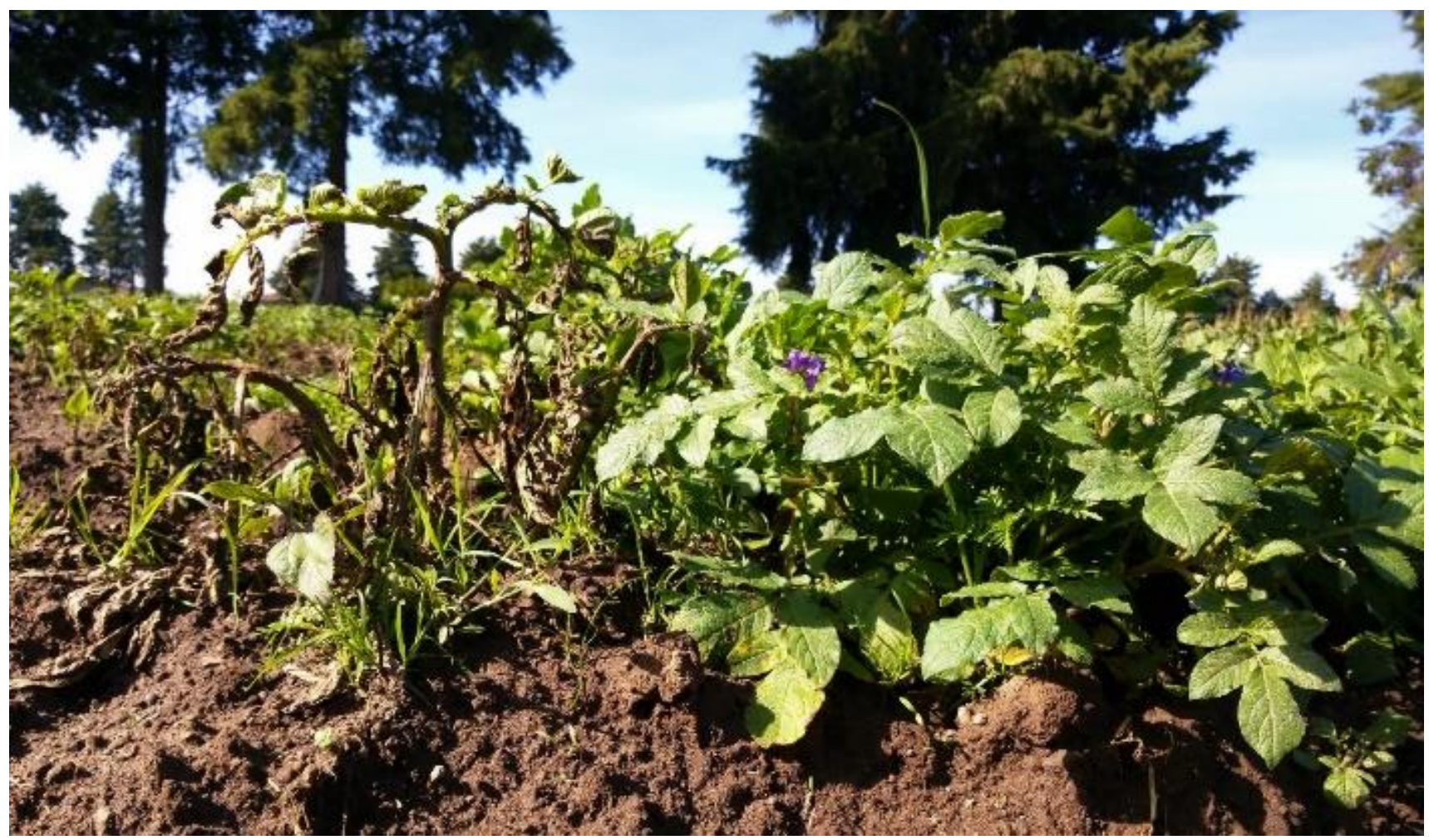

Figura 3. Comparación entre planta de Solanum tuberosum, susceptible al tizón tardío (izquierda) y una planta resistente de Solanum demissum (derecha) en el Valle de Toluca, México, 2019. 


\section{Las papas silvestres en mejoramiento} genético. Luego de la hambruna irlandesa se realizaron las primeras cruzas intraespecíficas de $S$. tuberosum que no originaron materiales resistentes al tizón tardío debido a que la base genética de las variedades europeas era muy estrecha en ese entonces (Zoteyeva et al., 2012). Además, la mayoría de los mejoradores creían que cruzar variedades europeas con variedades de sudamericanas y mexicanas ayudaría a generar papas con mayor vigor que le permitirían resistir mejor las enfermedades. Recién a inicios del siglo XX se realizaron cruzas interespecíficas entre $S$. tuberosum y $S$. demissum, especie que contiene 11 genes $R$ de resistencia raza-específica (Figura 3) (Turner, 2005).

Entre 1920 y 1940, el mejoramiento genético se basó en la resistencia vertical, obtenida de $S$. demissum, contra razas específicas de $P$. infestans. En ese entonces existían pocos patotipos de $P$. infestans dispersos fuera de México. A partir de 1950 se combinó la resistencia vertical y horizontal de varias papas silvestres, obteniéndose variedades con resistencia moderada pero duradera (LozoyaSaldaña, 2011). Los materiales resistentes de la década de 1980 contenían una base genética estrecha debido a que se obtuvieron a partir de un limitado número de genotipos (Zoteyeva et al., 2012), lo que provocó que la resistencia fuera superada sistemáticamente por nuevas variantes del patógeno, limitando su uso agrícola (Tiwari et al., 2015).

Actualmente, los cultivares resistentes a $P$. infestans existentes no son suficientes para reducir las pérdidas de rendimiento, haciendo que la investigación se centre en utilizar genes de resistencia de papas silvestres para obtener cultivares resistentes y duraderos contra un amplio rango de aislados del patógeno (Tiwari et al., 2013).

Existen unas 107 especies de papa silvestre, de ploidía variada y distribuidas en 16 países de América (Spooner et al., 2019). En la búsqueda de resistencia al tizón tardío, Pérez et al. (2000) identificaron 80 de 133 accesiones con resistencia cuantitativa; Micheletto et al. (2000) identificaron fuentes de resistencia en Solanum commersonnii y Solanum chacoense; Gopal \& Singh (2003) identificaron 10 materiales resistentes de Solanum tuberosum dentro una colección de 270 accesiones; Khiutti et al. (2015) observaron variación de la resistencia intra e interespecífica en 34 especies; Bachmann-Pfabe et al., (2019) identificaron 69 accesiones muy resistentes y 311 moderadamente resistentes; Karki et al. (2021) detectaron 68 especies resistentes al tizón tardío, indicando la presencia de genes $R B$; y Enciso-Maldonado et al., (2021) encontraron más de 20 accesiones con moderada y alta resistencia en el Valle de Toluca (Figura 4). Por su parte, el Instituto de Investigación de Plantas N. I. Vavilov (Rusia), almacena una de las más antiguas colecciones de papas silvestres del mundo y mediante el Proyecto CEEM (CornellEuropa del Este-México), en colaboración con el Centro de Investigación de Młochów (Polonia), se busca rescatar y guardar ese material genético para asegurar su disponibilidad para programas de mejoramiento en todo el mundo (Zoteyeva et al., 2012). Por su parte, el Banco de Germoplasma de Papa del Departamento de Agricultura de los Estados Unidos (USDA/ARS - U.S. Potato Genebank) cuenta con base de datos libre del. 

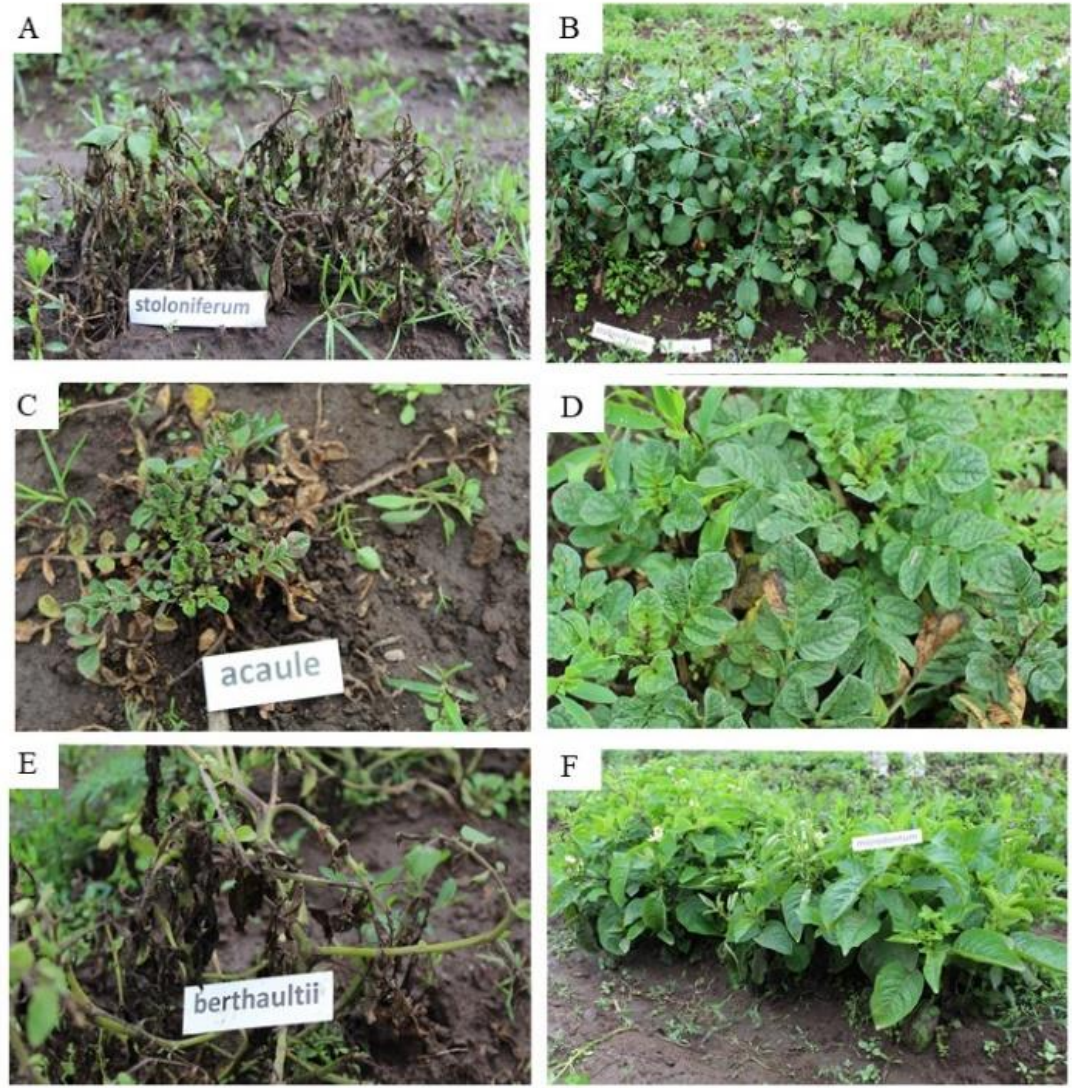

Figura 4. Variabilidad de la resistencia en accesiones dentro de una misma especie y entre especies. A) Solanum stoloniferum susceptible (A) y resistente (B); Solanum acaule susceptible (C) y resistente (D), accesión de Solanum brevicaule susceptible (E) y accesión de resistente (F) en el Valle de Toluca, México, 2020.

USDA Germplasm Resources Information Network (GRIN) (2021) en donde reportan más de 25 especies resistentes Similarmente, el Centro Internacional de la Papa (Perú) conserva y provee materiales para el resto del mundo y constantemente buscan fuentes de resistencia al tizón tardío (Lindqvist-Kreuze et al., 2020).

\section{Desafíos y avances en el uso de papas} silvestres. Existen barreras biológicas entre especies que limitan el aprovechamiento de los genes de resistencia de las papas silvestres. La hibridación y las cruzas interespecíficas se dificultan por la variabilidad de ploidías y número de balance del endospermo (NBE) entre la especie cultivada y las silvestres (Haverkort \& Kempenaar, 2016). El NBE se refiere al desarrollo del endospermo después de la fertilización y en papas se establece que la proporción de contribución genómica debe ser 2:1 (materno/paterno) para el desarrollo normal del endospermo (Depetris et al., 2018; Johnston et al., 1980). Para superar estas barreras se cultivan anteras y regeneran plantas diploides a partir de tetraploides, o haploides a partir de diploides (Pacheco-Sánchez et al., 2003), se realiza cruce de puentes entre especies, rescate de embriones, fusión de protoplastos o duplicación de cromosomas con posterior selección y retrocruzas normales con el material parental deseado (Bethke et al., 2017).

La minería de alelos y estudios de asociación del genoma completo han permitido explorar el genoma de papas silvestres para detectar genes homólogos 
de resistencia (Vossen et al., 2016; Tiwari et al., 2015; Gebhardt et al., 2004; Kim et al., 2012; Sokolova et al., 2011). Por otro lado, la construcción de mapas de ligamiento sirvió para desarrollar marcadores moleculares vinculados a los loci de resistencia (Tiwari et al., 2013).

Actualmente, existen varios marcadores ligados a genes $R$, loci de carácter cuantitativo $\mathrm{y}$ marcadores de polimorfismo de nucleótido simple (SNPs) que confieren resistencia al tizón tardío (Santa et al., 2018; Lindqvist-Kreuze et al., 2014).

Algunas especies en las que se han reportado genes de resistencia al tizón tardío son $S$. demissum; S. bulbocastanum; S. brachistotrichum, S. edinense; S. hjertingii, S. microdontum; S. schenckii; S. verrucosum, S. pinnatisectum, S. stoloniferum, S. papita; S. polytrichon; S. mochiquense, S. phureja; S. venturii; S. dulcamara, S. berthaultii; S. avilesi; S. capsicibaccatum, entre otras (Tiwari et al., 2013). Hasta la fecha, el gen $R B$ es el gen más eficiente para suprimir la infección por $P$. infestans (Lokossou et al., 2009; Park et al., 2005; Song et al., 2003), y sirvió para la generación de nuevas variedades con resistencia horizontal (Van der Vossen et al., 2003).

Posteriormente, también se encontraron homólogos del gen $R B$ en otras especies de Solanum (Wang et al., 2008). Con base en ensayos genéticos, de resistencia y generación de transgénicos, se han identificado genes $R B$ que son funcionalmente equivalentes a los de $S$. bulbocastanum en S. stoloniferum y S. verrucosum (Wang et al., 2008).

Uno de los mayores logros en el mejoramiento genético para combatir al tizón tardío fue el apilamiento de tres genes de resistencia al tizón tardío ( $R B, R p i-b l b 2$ y $R p i$-vnt1.1) en las variedades de papa "Desiree" y "Victoria" por medio de transgénesis, las cuales completaron sus ciclos mostrándose inmunes al tizón tardío y sin recibir aplicaciones de fungicida, mientras que las mismas variedades no transgénicas se destruyeron rápidamente (Ghislain et al., 2019).

Identificación de fuentes de resistencia. Para identificar materiales resistentes al tizón tardío se aplican distintas metodologías que incluyen pruebas de campo y ensayos de inoculación del patógeno en plantas enteras, en hojas desprendidas y ensayos in vitro (Forbes et al., 2005). Pérez et al. (2000) mencionan que los ensayos in vitro ofrecen una visión general de los niveles de resistencia en los germoplasmas y que son necesarias posteriores evaluaciones a campo. Por su parte, Karki et al. (2021) mencionan que los ensayos de hojas desprendidas ofrecen condiciones muy favorables para el desarrollo de la enfermedad, impidiendo la identificación precisa de la resistencia cuantitativa, y hacen que un mayor número de genotipos califique como susceptible; además, no siempre existe una relación cercana en la resistencia del follaje evaluada en laboratorio y campo (Lal et al., 2013). Las pruebas de campo que miden el área bajo la curva de progreso de la enfermedad proporcionan la mejor estimación de la resistencia a las enfermedades (Fry, 1978), sin embargo, en la mayoría de los sitios de evaluación es necesario realizar inoculaciones y cuando las condiciones no son favorables, los ensayos no son fiables debido a que el patógeno no es capaz de causar (Sieber et al., 2015). El Valle de Toluca en México es un sitio de importancia internacional para la evaluación de genotipos debido a que allí no se requieren inoculaciones artificiales debido a la gran cantidad de oosporas de cepas variables y virulentas que 
sobreviven al invierno (Lozoya-Saldaña, 2005;

Fernández-Pavía et al., 2004; Shakya et al., 2018).Estas condiciones hacen que cualquier clon de papa resistente al tizón tardío en Toluca tenga muchas posibilidades de seguir siéndolo en el resto del mundo (Lozoya-Saldaña, 2005).

\section{CONCLUSIÓN}

La búsqueda de resistencia al tizón tardío en papas silvestres es una parte importante dentro del mejoramiento genético de la papa debido a que $P$. infestans ha vencido por años la resistencia genética de la planta.

La utilización de los genes de resistencia de las papas silvestres todavía es limitada debido a las barreras a nivel de ploidía y la incompatibilidad del número del balance del endospermo con la papa cultivada. El mejoramiento genético asistido por marcadores moleculares y las nuevas técnicas de modificación genética para transferir o apilar rápidamente genes de resistencia de papas silvestres a cultivares de papa son una herramienta con gran potencial para acelerar los procesos de obtención de genotipos de interés.

El Valle de Toluca en México es un sitio ideal para realizar trabajos de identificación de fuentes de resistencia e impulsar programas de mejoramiento genético ya que cuenta con la ventaja de ser el mayor centro de diversidad del $P$. infestans, con clima ideal para el patógeno y con gran variabilidad de resistencia genética en las papas silvestres que se distribuyen naturalmente en la zona.

\section{AGRADECIMIENTO}

Los autores reconocen y agradecen al Consejo Nacional de Ciencia y Tecnología (CONACYT, México) por otorgar las becas de posgrado a GAEM, GDG y ALS, al apoyo de K. Raman y J. Bamberg, colaboradores del Proyecto CEEM (Cornell Eastern Europe-Mexico) sobre Control de Tizón Tardío y al Lic. Biól. Bruno Olmedo de la Universidad Nacional de Asunción por las ilustraciones.

Declaración de interés. Los autores declaran no tener conflicto de intereses.

\section{REFERENCIAS BIBLIOGRÁFICAS}

Bethke, P. C., Halterman, D. A. \& Jansky, S. (2017). Are we getting better at using wild potato species in light of new tools. Crop Science, 57 (3), 1241-1258. https://doi.org/10.2135/cropsci2016.10.0889

Burgos, G., Zum Felde, T., Andre, C. \& Kubow, S. (2020). The potato and its contribution to the human diet and health. In The potato crop, pp. 37-74. Springer, Cham. https://doi.org/10.1007/978-3-030-28683-5_2

Corredor-Moreno, P. \& Saunders, D. G. (2020). Expecting the unexpected: factors influencing the emergence of fungal and oomycete plant pathogens. New Phytologist, 225 (1), 118-125. https://doi.org/10.1111/nph.16007

Depetris, M. B., Acuña, C. A., Pozzi, F. I., Quarin, C. L. \& Felitti, S. A. (2018). Identification of genes related to endosperm balance number insensitivity in Paspalum $\begin{array}{llll}\text { notatum. Crop } & \text { Science, } 58 & \text { (2), 813-822. }\end{array}$ https://doi.org/10.2135/cropsci2017.04.0260

Douches, D. S., Kirk, W. W., Bertram, M. A., Coombs, J. J. \& Niemira, B. A. (2002). Foliar and tuber assessment of late blight (Phytophthora infestans (Mont.) de Bary) reaction in cultivated potato (Solanum tuberosum L.). Potato research, $45 \quad$ (2-4), 215-224. https://doi.org/10.1007/BF02736116

Devaux, A., Goffart, J. P., Petsakos, A., Kromann, P., Gatto, M., Okello, J., Suarez, V. \& Hareau, G. (2020). Global food security, contributions from sustainable potato agrifood systems. In The potato crop (pp. 3-35). Springer, Cham. https://doi. org/10.1007/978-3-030-28683-5_1

Enciso-Maldonado, G. A., Lozoya-Saldana, H. Colinas-Leon, M. T., Cuevas-Sanchez, J. A. Sanabria-Velázquez, A. D. Bamberg, J. \& Raman, K. V. (2021). Assessment of wild Solanum species for resistance to Phytophthora infestans (Mont.) de Bary in the Toluca Valley, Mexico. [en prensa] https://doi.org/10.1007/s12230-021-09856-x

FAOSTAT. (2021). United Nations Food and Agricultural 
Organization.

Disponible

http://www.fao.org/faostat/en/\#data

15.08.2021)

Fernández-Pavía, S. P., Grünwald, N. J., Diaz-Valasis, M., Cadena-Hinojosa, M. \& Fry, W. E. (2004). Soilborne oospores of Phytophthora infestans in central Mexico survive winter fallow and infect potato plants in the field. Plant Disease, $88 \quad$ (1), 29-33. https://doi.org/10.1094/PDIS.2004.88.1.29

Flier, W. G., Grünwald, N. J., Kroon, L. P. N. M., Sturbaum, A. K., Van den Bosch, T. B., Garay-Serrano, E., LozoyaSaldaña, H., Fry, W. F. \& Turkensteen, L. J. (2003). The population structure of Phytophthora infestans from the Toluca Valley of Central Mexico suggests genetic differentiation between populations from cultivated potato and wild Solanum spp. Phytopathology, 93 (4), 382-390. https://doi.org/10.1094/PHYTO.2003.93.4.382

Foolad, M. R., Merk, H. L. \& Ashrafi, H. (2008). Genetics, genomics and breeding of late blight and early blight resistance in tomato. Critical Reviews in Plant Sciences, 27(2),

75-107. https://doi.org/10.1080/07352680802147353

Forbes, G. A., Chacón, M. G., Kirk, H. G., Huarte, M. A., Van Damme, M., Distel, S., ... \& Capezio, S. (2005). Stability of resistance to Phytophthora infestans in potato: an international evaluation. Plant pathology, 54 (3), 364372. https://doi.org/10.1094/PHYTO-01-15-0005-FI

Fry, W. E., Birch, P. R. J., Judelson, H. S., Grünwald, N. J., Danies, G., Everts, K. L., Gevens, A. J. , Gugino, B. K., Johnson, D. A., Johnson, S. B., McGrath, M. T., Myers, K. L., Ristaino, J. B., Roberts, P. D., Secor, G. \& Smart, C. D. (2015a). Five reasons to consider Phytophthora infestans a reemerging pathogen. Phytopathology, 105 (7), 966-981. https://doi.org/10.1111/j.13653059.2005.01187.x

Fry, B., Danies, G., Small, I. \& Myers, K. (2015b). Recent developments concerning the population biology and control strategies of Phytophthora infestans in the USA. PPO-Special Report, 17, 45-50. https://agro.au.dk/fileadmin/euroblight/Workshops/Proce edings/Special_Report_17_Totaal_LR.pdf\#page=47

Fry, W. E. \& Grünwald, N. J. (2010). Introduction to oomycetes. The Plant Health Instructor. doi:10.1094/PHI-I-2012-0220-01

Fry, W. E. \& Goodwin, S. B. (1997). Resurgence of the Irish potato famine fungus. Bioscience, 47 (6), 363-371. https://doi.org/10.2307/1313151

Gebhardt, C., Ballvora, A., Walkemeier, B., Oberhagemann, P. \& Schüler, K. (2004). Assessing genetic potential in germplasm collections of crop plants by marker-trait association: a case study for potatoes with quantitative variation of resistance to late blight and maturity type. Molecular Breeding, 13 (1), 93-102. https://doi.org/10.1023/B:MOLB.0000012878.89855.df

Ghislain, M., Byarugaba, A. A., Magembe, E., Njoroge, A., Rivera, C., Román, M. L., Tovar, J. C., Gamboa, S., Forbes, G. A., Kreuze, J. F., Barekye, A. \& Kiggundu, A. (2019). Stacking three late blight resistance genes from wild species directly into African highland potato varieties confers complete field resistance to local blight races. Plant Biotechnology Journal, 17(6), 1119-1129. https://doi.org/10.1111/pbi.13042

Schepers, H., Hausladen, H., Grønbech Hansen, J., Nielsen, B., Abuley, I., Andersson, B., Liljeroth, E., Edin, E., Bain, R., Ritchie, F., Gaucher, D., Ivanovic, Z., Blagojevic, J., Kildea, S., Filipov, A., Kuznetsova, M., Hannukkala, A., Eikemo, H., Nærstad, R.,..., Vanhaverbeke, P. (2015).
Epidemics and control of early \& late blight, 2013 \& 2014 in Europe. In Proceedings of the fifteenth EuroBlight workshop, Brasov, Romania, 13-15 May 2015/Ed. HTAM Schepers. Applied Plant Research, Wageningen UR. https://euroblight.net//fileadmin/euroblight/Workshops/ AArhus/Proceedings/Special_Report_18_Totaal.pdf

Grünwald, N. J. \& Flier, W. G. (2005). The biology of Phytophthora infestans at its center of origin. Annual review of phytopathology, 43, 171-190. https://doi.org/10.1146/annurev.phyto.43.040204.13590 6

Halterman, D. A., Kramer, L. C., Wielgus, S. \& Jiang, J. (2008). Performance of transgenic potato containing the late blight resistance gene RB. Plant disease, 92 (3), 339-343. https://doi.org/10.1094/PDIS-92-3-0339

Haverkort, A. J., Boonekamp, P. M., Hutten, R., Jacobsen, E., Lotz, L. A. P., Kessel, G. J. T., Visser, R. G. G. F. \& Van der Vossen, E. A. G. (2008). Societal costs of late blight in potato and prospects of durable resistance through cisgenic modification. Potato research, 51 (1), 47-57. https://doi.org/10.1007/s11540-008-9089-y

Haverkort, A. J. \& Kempenaar, C. (2016). Recent advances in biotechnology and information technology in the potato industry. In The Dundee Conference, pp. 183-190. https://library.wur.nl/WebQuery/wurpubs/fulltext/41060 5

Johnston, S. A., Den Nijs, T. P. M., Peloquin, S. J. \& Hanneman, R. E. (1980). The significance of genic balance to endosperm development in interspecific crosses. Theoretical and applied genetics, 57 (1), 5-9. https://doi.org/10.1007/BF00276002

Karki, H. S., Jansky, S. H. \& Halterman, D. A. (2021). Screening of wild potatoes identifies new sources of late blight resistance. Plant Disease, 105 (2), 368-376. https://doi.org/10.1094/PDIS-06-20-1367-RE

Kim, H. J., Lee, H. R., Jo, K. R., Mortazavian, S. M., Huigen, D. J., Evenhuis, B., Kessel, G., Visser, R. G. F., Jacobsen, E. \& Vossen, J. H. (2012). Broad spectrum late blight resistance in potato differential set plants MaR8 and MaR9 is conferred by multiple stacked $\mathrm{R}$ genes. Theoretical and applied genetics, 124 (5), 923935. https://doi.org/10.1007/s00122-011-1757-7

Kirk, W. W., Felcher, K. J., Douches, D. S., Niemira, B. A. \& Hammerschmidt, R. (2001). Susceptibility of potato (Solanum tuberosum L.) foliage and tubers to the US8 genotype of Phytophthora infestans. American Journal of Potato Research, 78 (4), 319-322. https://doi.org/10.1007/BF02875697

Lal, M., Luthra, S. K., Singh, B. P. \& Yadav, S. (2013). Screening of genotypes against potato late blight. Potato Journal, 40 http://epubs.icar.org.in/ejournal/index.php/PotatoJ/article /view/31811

Leesutthiphonchai, W., Vu, A. L., Ah-Fong, A. M. \& Judelson, H. S. (2018). How does Phytophthora infestans evade control efforts? Modern insight into the late blight disease. Phytopathology, $108 \quad$ (8), 916-924. https://doi.org/10.1094/PHYTO-04-18-0130-IA

Lindqvist-Kreuze, H., Gamboa, S., Izarra, M., Pérez, W., Correa, M. Y., Astete, A., Särkinen, T., Cueva, P. \& Gonzáles, P. (2020). Population structure and host range of the potato late blight pathogen Phytophthora infestans in Peru spanning two decades. Plant Pathology, 69 (2), 334-346. https://doi.org/10.1111/ppa.13125

Lindqvist-Kreuze, H., Gastelo, M., Perez, W., Forbes, G. A., de Koeyer, D. \& Bonierbale, M. (2014). Phenotypic 
stability and genome-wide association study of late blight resistance in potato genotypes adapted to the tropical highlands. Phytopathology, 104 (6), 624-633. https://doi.org/10.1094/PHYTO-10-13-0270-R

Lokossou, A. A., Park, T. H., van Arkel, G., Arens, M., RuyterSpira, C., Morales, J., Whisson, S. C., Birch P. R. J., Visser, R. G. F., Jacobsen, E., \& van der Vossen, E. A. (2009). Exploiting knowledge of R/Avr genes to rapidly clone a new LZ-NBS-LRR family of late blight resistance genes from potato linkage group IV. Molecular plant-microbe interactions, 22 (6), 630641. https://doi.org/10.1094/MPMI-22-6-0630

Lozoya-Saldaña, H. (2005). Importancia Internacional del Valle de Toluca, México, en estudios sobre el tizón tardío de la papa (Solatium tuberosum L.), causado por Phytophthora infestans (Mont.) de Bary. Revista Mexicana de Fitopatología, 23 (3), 312-319.

Lozoya-Saldana, H. (2011). Evolution of vertical and horizontal resistance and its application in breeding resistance to potato late blight. Potato Journal, 38 (1), 18.

http://epubs.icar.org.in/ejournal/index.php/PotatoJ/article /view/32385

Mabon, R., Guibert, M., Corbière, R. \& Andrivon, D. (2021). An improved PCR method for rapid and accurate identification of mating types in the late blight pathogen Phytophthora infestans. Plant Health Progress, PHP-02. https://doi.org/10.1094/PHP-02-21-0026-FI

Mayton, H., Griffiths, H., Simko, I., Cheng, S., Lorenzen, J., De Jong, W. \& Fry, W. E. (2010). Foliar and tuber late blight resistance in a Solanum tuberosum breeding population. Plant breeding, $129 \quad$ (2), 197-201. https://doi.org/10.1111/j.1439-0523.2009.01671.x

Micheletto, S., Boland, R. \& Huarte, M. (2000). Argentinian wild diploid Solanum species as sources of quantitative late blight resistance. Theoretical and Applied Genetics, $101 \quad$ (5-6), 902-906. https://doi.org/10.1007/s001220051560

Pacheco-Sánchez, M., Lozoya-Saldaña, H. \& Colinas-León, M. T. (2003). Reguladores de crecimiento y pretratamiento con frío en la androgénesis in vitro de solanum iopetalum 1. Agrociencia, $37 \quad$ (3), 257-265. https://www.redalyc.org/pdf/302/30237305.pdf

Park, T. H., Vleeshouwers, V. G., Kim, J. B., Hutten, R. C. \& Visser, R. G. (2005). Dissection of foliage and tuber late blight resistance in mapping populations of potato. Euphytica, $143 \quad$ (1), 75-83. https://doi.org/10.1007/s10681-005-2658-0

Pérez, W., Salas, A., Raymundo, R., Huaman, Z., Nelson, R. \& Bonierbale, M. (2000). Evaluation of wild potato species for resistance to late blight. Scientist and Farmer, Partners in Research for the 21st Century, Program Report 1999-2000, 49-62. https://citeseerx.ist.psu.edu/viewdoc/download?doi=10.1 $.1 .527 .6319 \&$ rep=rep1\&type $=$ pdf

Qi, J., Asano, T., Jinno, M., Matsui, K., Atsumi, K., Sakagami, Y., \& Ojika, M. (2005). Characterization of a Phytophthora mating hormone. Science, 309 (5742), 1828-1828. DOI: 10.1126/science. 1114756

Santa, J. D., Berdugo-Cely, J., Cely-Pardo, L., Soto-Suárez, M., Mosquera, T. \& Galeano M, C. H. (2018). QTL analysis reveals quantitative resistant loci for Phytophthora infestans and Tecia solanivora in tetraploid potato (Solanum tuberosum L.). Plos one, 13 (7), e0199716. https://doi.org/10.1371/journal.pone.0199716

Shakya, S. K., Larsen, M. M., Cuenca-Condoy, M. M., LozoyaSaldaña, H. \& Grünwald, N. J. (2018). Variation in genetic diversity of Phytophthora infestans populations in Mexico from the center of origin outwards. Plant disease, 102 (8), 1534-1540. https://doi.org/10.1094/PDIS-11-17-1801-RE

Sieber, K., Forster, G. M., Berger, A., Hammann, T., Kellermann, A. \& Schwarzfischer, A. (2015). Assessment of genetic hotspots for Phytophthora resistance and their use as molecular markers in potato breeding. PPO-Special Report no. 17, 57. https://euroblight.net/fileadmin/euroblight/Publications/S pecial_Report_17_Totaal_LR.pdf\#page=59

Sokolova, E., Pankin, A., Beketova, M., Kuznetsova, M., Spiglazova, S., Rogozina, E. \& Khavkin, E. (2011). SCAR markers of the R-genes and germplasm of wild Solanum species for breeding late blight-resistant potato cultivars. Plant Genetic Resources, 9 (2), 309. https://doi.org/10.1017/S1479262111000347

Song, J., Bradeen, J. M., Naess, S. K., Raasch, J. A., Wielgus, S. M., Haberlach, G. T., Liu, J., Kuang, H., AustinPhillips, S., Buell, C. R., Helgeson, J. P. \& Jiang, J. (2003). Gene RB cloned from Solanum bulbocastanum confers broad spectrum resistance to potato late blight. Proceedings of the national academy of sciences, $100 \quad$ (16), 9128-9133. https://doi.org/10.1073/pnas.1533501100

Spooner, D. M., Jansky, S., Rodríguez, F., Simon, R., Ames, M., Fajardo, D. \& Castillo, R. O. (2019). Taxonomy of wild potatoes in northern South America (Solanum section Petota). Systematic Botany Monographs, 108, 1305. https://doi.org/10.2307/25027915

Stokstad, E. (2019). The new potato. Science, 363 (6427), 574577

Tiwari, J. K., Siddappa, S., Singh, B. P., Kaushik, S. K., Chakrabarti, S. K., Bhardwaj, V. \& Chandel, P. (2013). Molecular markers for late blight resistance breeding of potato: an update. Plant Breeding, 132 (3), 237-245. https://doi.org/10.1111/pbr.12053

Tiwari, J. K., Devi, S., Sharma, S., Chandel, P., Rawat, S. \& Singh, B. P. (2015). Allele mining in Solanum germplasm: cloning and characterization of RBhomologous gene fragments from late blight resistant wild potato species. Plant molecular biology reporter, $33 \quad$ (5), 1584-1598. https://doi.org/10.1007/s11105-015-0859-9

Turner, R. S. (2005). After the famine: Plant pathology, Phytophthora infestans, and the late blight of potatoes, 1845-1960. Historical Studies in the Physical and Biological Sciences, $35 \quad$ (2), 341-370. https://doi.org/10.1525/hsps.2005.35.2.341

USDA. (2021). Red de información sobre recursos de germoplasma (GRIN). Servicio de Investigaciones Agropecuarias. Departamento de Agricultura de EE.EE. https://www.ars-grin.gov/

Vleeshouwers, V. G., Raffaele, S., Vossen, J. H., Champouret, N., Oliva, R., Segretin, M. E., Rietman, H., Cano, L. M., Lokossou, A., Kessel, G., Pel, M. A. \& Kamoun, S. (2011). Understanding and exploiting late blight resistance in the age of effectors. Annual review of phytopathology, 49, 507-531. https://doi.org/10.1146/annurev-phyto-072910-095326

Van Der Vossen, E., Sikkema, A., Hekkert, B. T. L., Gros, J., Stevens, P., Muskens, M., Wouters, D., Pereira, A., Stiekema, W. \& Allefs, S. (2003). An ancient R gene from the wild potato species Solanum bulbocastanum confers broad-spectrum resistance to Phytophthora infestans in cultivated potato and tomato. The plant journal, 36 (6), 867-882. https://doi.org/10.1046/j.1365- 
313X.2003.01934.x

Vossen, J. H., van Arkel, G., Bergervoet, M., Jo, K. R., Jacobsen, E., \& Visser, R. G. (2016). The Solanum demissum R8 late blight resistance gene is an Sw-5 homologue that has been deployed worldwide in late blight resistant varieties. Theoretical and Applied Genetics, 129(9), $1785-1796$ https://doi.org/10.1007/s00122-016-2740-0

Wang, J., Fernández-Pavía, S. P., Larsen, M. M., GaraySerrano, E., Gregorio Cipriano, R., Rodríguez-Alvarado, G., Grünwald, N. J. \& Goss, E. M. (2017). High levels of diversity and population structure in the potato late blight pathogen at the Mexico centre of origin. Molecular Ecology, 26, 1091-1107. https://doi.org/10.1111/mec.14000
Zoteyeva, N., Chrzanowska, M., Flis, B. \& ZimnochGuzowska, E. (2012). Resistance to pathogens of the potato accessions from the collection of NI Vavilov Institute of Plant Industry (VIR). American journal of potato research, 89 (4), 277-293. https://doi.org/10.1007/s12230-012-9252-5

Zoteyeva, N. M. (2020). Late blight resistance of wild potato species under field conditions in the Northwest of Russia. Proceedings on applied botany, genetics and breeding, $180 \quad$ (4), 159-169. https://doi.org/10.30901/2227-8834-2019-4-159-169 